\title{
AN EXTENDED COMPLEX KALMAN FILTER FOR FREQUENCY MEASUREMENT OF DISTORTED SIGNALS
}

\author{
P. K. Dash* \\ G. Panda \\ A.K. Pradhan \\ A. Routray"* \\ B. Duttagupta"* \\ * Regional Engineering College, Rourkela - 769008, India \\ ** Indian Institute of Technology, Kharagpur, India
}

\section{ABSTRACT}

The design of an extended complex Kalman filter for the measurement of power system frequency has been presented in this paper. The design principles and the validity of the model has been outlined. A complex model has been developed to track a distorted signal that belongs to a power system. The model inherently takes care of the frequency measurement along with the amplitude and phase of the signals. The theory has been applied to standard signals representilig the worst-case measurement and network conditions in a typical power system. The proposed algorithm is suitable for realtime applications where the measurement noise and other disturbances are high. The complex quantities can be conveniently handled using a floating point processor. Comparison of the results of the proposed method with those obtained from a real extended Kalman filter reveal the superior performance of the former method.

\section{INTRODUCTION}

In this paper the method presentde deals with the measurement of the parameters of a power system signal which is usually contaminated with noise and ligh disturbances. The amplitude and phase estimation of a digitized signal has been important area of research for past several years and the methods have been almost standardized for signals with known frequencies. However, if the frequency is not known apriori it becomes a formidable task to accurately measure the amplitude and phase. It has been a perpetual problem even now to correctly estimate the frequency of an incoming signal from its sampled values under high noise conditions. The reason for this is the association of severe non-linearity in the modeling process. On the other hand the philosophy of protection for power systems has been undergoing a metamorphosis. The modern protective relays employ sophisticated signal processing algorithms to accurately estimate the system conditions from the measured voltage and current signals. The pitfalls in these schemes stem from the frequency measurement algorithm which under transient and abnormal conditions cannot sense the correct value.

Many algorithms have been reported in the literature for measurement and estimation of frequency [1,2,3,4]. A comparative study among four different trackers has been outlined in [5]. In this paper the performance of (i) an adaptive notch filter (ii) a multiple frequency tracker (iii) an adaptive IIR filter and an (iv) hyperstabel adaptive line enhancer have been presented. The complex Kalman filtering has been used in [6] to estimate the frequency of the signals corrupted with white noise. The present paper is based on the fundamental work carried out in [6]. However, in practice the real and imaginary signals can not be obtained simultaneously. Therefore, keeping in view the measurement of power system signals the signal model has been modified. The output equation is rewritten to calculate the real signal.

\section{II.SIGNAL MODEL}

The non-linear state space description of the power system signal can have various forms. Numerous linear as well as non-linear models have been proposed to estimate the amplitude, phase and frequency of a single sinusoid. This signal can also represented by a complex model. With availability of floating point DSP processors the computation in complex domain is no longer a difficult task. Moreover, the complex representation is much simpler and direct as far as the frequency measurement is concerned. Once the signal model is established the Extended Klaman Filter theory [7] can be applied to identify the filter equations and computational steps.

Let an observation signal $y_{k}$ at time $t_{k}$ be a sum of $z_{k}$ of $M$ sinsoids with additive noise $v_{k}$

$$
\begin{aligned}
& y_{k}=z_{k}+v_{k} \quad k=1,2,3 \ldots . N \\
& \text { where, } z_{k}=\sum_{i=1}^{M} a_{i} \sin \left(\omega_{i}{ }_{k}+\phi_{i}\right) \\
& \omega_{i}=2 \pi f_{i}, t_{k}=k \Delta t
\end{aligned}
$$

in which $a_{h} f_{1}$ and $\phi_{1}$ are amplitude, frequency and the phase of the $i^{\text {th }}$ sinusoids respectively. The observation noise $v_{k}$ is a Gaussian white noise with zero-mean and variance $\sigma_{v}{ }^{2}$.

In case of power system signals the percentage of frequency components other than thefundamental is low. Therefore these harmonic components need not be considreed in the model.

For such systems equation (2) reduces to a single sinusoid of the form $y_{k}=a_{1} \sin \left(k \omega T_{s}+\phi_{1}\right)$

$T_{s}=$ sampling time, $\omega_{l}=$ the fundamental angular frequency.

The observation signal can be represented in an autoregressive complex form as follows:

$\left[\begin{array}{c}\alpha \\ u_{k+1} \\ u_{k+1}^{*}\end{array}\right]=\left[\begin{array}{ccc}1 & 0 & 0 \\ 0 & \alpha & 0 \\ 0 & 0 & 1 / \alpha\end{array}\right]\left[\begin{array}{c}\alpha \\ u_{k} \\ u_{k}\end{array}\right]$ 


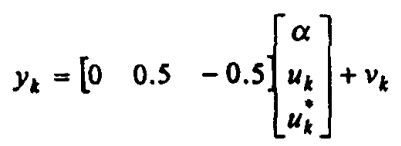

where, $\alpha=\exp \left(j \omega_{1} T_{s}\right)$

$u_{k}=a_{1} \exp \left(j k w_{1} T_{s}+j \phi_{1}\right)$

$u_{k}{ }_{k}=a_{1} \exp \left(-j k w_{1} T_{s}-j \phi_{1}\right)$

$v_{k}=$ measurement noise

The above non-linear process can be represented as:

$x_{k+1}=f\left(x_{k}\right)$

$y_{k+1}=H x_{k}+v_{k}$

where $x(k)=\left[\begin{array}{lll}\alpha & u_{k} & u_{k}^{*}\end{array}\right]^{T}$

$f\left(x_{k}\right)=\left[\begin{array}{lll}\alpha & \alpha u_{k} & u_{k}^{*}\end{array} / \alpha\right]^{T}$

$H=\left[\begin{array}{lll}0 & 0.5 & 0.5\end{array}\right]$

Linearising the above system and applying the extended complex Kalman filter to the first order system, a non-linear recursive filter for estimating a single complex sinusoid and its frequency in white noise is obtained as follows [6]:

$$
\begin{aligned}
& \hat{x}_{k / k}=\hat{x}_{k / k-1}+K_{k}\left(y_{k}-H \hat{x}_{k / k-1}\right) \\
& \hat{x}_{k+1 / k}=f\left(\hat{x}_{k / k}\right) \\
& K_{k}=\hat{P}_{k / k-1} H^{* T}\left[H \hat{P}_{k / k-1} H^{* T}+1\right]^{-1} \\
& \hat{P}_{k / k}=\hat{P}_{k / k-1}-K_{k} H \hat{P}_{k / k-1} \\
& \hat{P}_{k / k+1}=F_{k} \hat{P}_{k / k} F_{k}^{* T} \\
& \text { where, }
\end{aligned}
$$

$$
\begin{aligned}
& F_{k}=\left.\frac{\partial f\left(x_{k}\right)}{\partial x_{k}}\right|_{x_{k}=\hat{x}_{k / k}}=\left[\begin{array}{ccc}
1 & 0 & 0 \\
\hat{x}_{k / k}(2) & \hat{x}_{k / k}(1) & 0 \\
\frac{-\hat{x}_{k / k}(3)}{\hat{x}_{k / k}^{2}(1)} & 0 & \frac{1}{\hat{x}_{k / k}(1)}
\end{array}\right] \\
& \sum_{k+1 / k}=E\left\{\left(x_{k+1}-\hat{x}_{k+1 / k}\right)\left(x_{k+1}-\hat{x}_{k+1 / k}\right)^{* T}\right\} \\
& \dot{x}_{1 / 0}=\bar{x}_{1} \quad \hat{P}_{1 / 0}=\hat{\Sigma}_{1 / 0} / \sigma_{v}^{2} \\
& \left.\hat{\Sigma}_{1 / 0}=\sum_{x !}, \quad \sum_{x 1}=E\left\{\left(x_{1}-\bar{x}_{1}\right) x_{1}-\bar{x}_{1}\right)^{\bullet T}\right\} \\
& \dot{P}_{k / k}=\dot{\Sigma}_{k / k} / \sigma_{v}^{2} \quad \hat{P}_{k+1 / k}=\dot{\Sigma}_{k+1 / k} / \sigma_{v}^{2} \\
& \dot{\Sigma}_{k / k}=E\left\{\left(x_{k}-\dot{x}_{k / k}\right)\left(x_{k}-\hat{x}_{k / k}\right)^{* T}\right\}
\end{aligned}
$$

The filter is non-linear and therefore the gain $K_{k}$ and the covariance matrix $\hat{P}_{k / k}$ depend on the estimate $\tilde{x}_{k / k}$ of the state vector $x_{k}$.

\section{III.SIMULATION}

The following tests have been performed to evaluate the performance of the above method.

Test Signal - I: Fundamental Signal with white noise [Signal to noise ratio (SNR) $13 \mathrm{~dB}$ ]

Signal $=A \cos \left(k \omega T_{s}+\phi\right)+$ Zero mean Gaussian while noise of standard deviation 0.1 where, $A=1.0$

Test Signal- II: Fundamental Signal with harmonics and white noise [SNR 37Db]
Signal $=A \cos \left(k \omega T_{s}+\phi\right)+A / 10 \cos \left(3 k \omega T_{s}+\phi\right)+A / 20$ $\cos \left(5 k \omega T_{s}+\phi\right)+$ noise as above with standard deviation 0.01 ; where, $A=1.0$

The problem with all Kalman filter algorithms is to reset the covariance matrix. After initial convergence the gain $K_{k}$ and the covariance matrix $P_{\mathrm{kk}}$ settle to very small values. Subsequently when some of the parameters (amplitude, phase and frequency) of the signal change the covariance matrix has to be reset to quickly track these values. In this paper a method based on the magnitude of the error has been used.

The decision to set the covariance matrix to the initial value is based on a hysteresis type decision block. The hysteresis band is determined by the amount of noise and the nature of convergence. If the noise is estimated to be around $10 \%$ of the amplitude then the hysteresis band is chosen to be $20-30 \%$ of the amplitude to avoid frequent resetting of the covariance matrix. A flag is set when the error exceeds the higher threshold $e_{11}$ and is reset when the error falls below the lower threshold $e_{L}$. If the flag is $l$ and any of the Kalman gains are very small then the covariance is reset and the flag ia also reset to 0 that there is no immediate resetting of the covariance matrix.

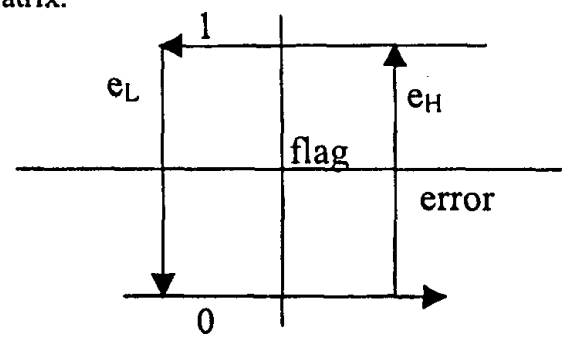

Fig 1 The hysterisıs band for resetting the covariance matrix

Generally the frequency variation in power systems is limited to $\pm 5 \mathrm{~Hz}$. Therefore, for faster tracking the frequency is limited to $40 \mathrm{~Hz}$ on the lower side and $60 \mathrm{~Hz}$ on the upper side. This results in the stable operation of the filter and does not lead the filter wayward and loose track of the desired frequency under high noise conditions.

Two different types of test signal as above have been taken to study the effectiveness of the proposed algorithm. In each of the cases the fundamental amplitude and frequency are subjected to sudden changes and under such conditions the tracking ability of the proposed filter is examined. The same tests are also carried out using an ordinary extended Kalman filter (Appendix). The comparative performance has been presented in Figs. 2 to7.

\section{A. Case I}

The amplitude of a sinusoidal power system signal is suddenly reduced from 1.4 p.u. to 1.0 p.u. and the instantaneous waveforms are displayed in Fig. 2. In another case, harmonics and more noise are added to the signal and its amplitude is suddenly reduced from 1.2 p.u. to 0.8 p.u. and Fig. 3 exhibits corresponding waveforms. The instantaneous waveforms have been displayed in Fig.2 The comparative study shows that the complex Kalman filter exhibits slightly inferior performance in estimating the amplitude and frequency in case of the signals without harmonics. Both the 
filters (real and complex) settle down at the new values within approximately half a cycle (about 0.01 second) of the fundamental time period. The proposed complex filter exhibits a little oscillation in the frequency during the amplitude change, whereas for the second type of signal (i.e. signal with harmonics) the-performance of the complex Kalman filter is unable to track the amplitude with 1.2 p.u. fundamental, 0.12 p.u. third harmonic and 0.06 p.u. fifth harmonic. However, as soon as the amplitudes of these components are reduced to $0.8,0.08$ and 0.04 respectively the filter settles down quickly.

\section{B. Case - II}

In this case the amplitude is suddenly increased from 1.0 p.u. to 1.4 p.u. for the ignal with harmonics. For the test signal II the real Kalman filter looses complete track of the signal with the new value of amplitude (Fig.4). Whereas, under identical conditions the complex Kalman filter takes almost 1-cycle (0.02 seconds) of the fundamental to track the amplitude, phase and frequency of the signal with the new amplitude.

\section{Case - III}

The frequency is suddenly increased from $50 \mathrm{~Hz}$ to $54 \mathrm{~Hz}$. The study shows that the complex Kalman filter exhibits quicker tracking performance as compared to the ordinary Kalman filter in case of the signal without harmonics (Fig.5). The proposed filter settles within 0.75 cycles $(0.15$ seconds) of the fundamental signal. But the real Kalman filter takes over 5 cycles. For the test signal - II, the later goes unstable after the frequency has been increased, whereas the proposed filter takes around 3-cycles to settle down at the new value of the frequency. These waveforms are depicted in Fig.6.

\section{Case - IV}

Fig. 8 shows the waveforms when the frequency is suddenly reduces from $50 \mathrm{~Hz}$. to $46 \mathrm{~Hz}$. For test signal - I the proposed filter takes around 0.015 seconds $(0.75$ cycles) to settle to the new value of the frequency. Whereas, the real filter is slower and takes almost one and half cycles $(0.03$ seconds) before settling at these values. Besides it is observed that the real filter malfunction and lose complete track of the signal when the signal is contaminated with harmonics and noise.

\section{CONCLUSION}

A non-linear complex Kalman filter has been proposed to estimate instantaneous frequency changes for a power system signal contaminated with noise and harmonics. The hysteresis method has been suggested for resetting the covariance matrix, which enables fast tracking of the filter and involves less computation. This makes the filter attractive for real time implementation. The issues such as stability of the algorithin and performance under other disturbance conditions are currently under investigation.

\section{APPENDIX}

Let the signal model as usual is represented by $z_{k}=a_{1} \sin \left(k w_{1} T_{s}+\phi_{1}\right)$ where $T_{s}=$ sampling time, $w_{l}=$ the fundamental angular frequency

The observation signal can be represented in a nonlinear state-space model as follows:

$\left[\begin{array}{l}x_{1(k+1)} \\ x_{2(k+1)} \\ x_{3(k+1)}\end{array}\right]=\left[\begin{array}{lll}1 & 0 & 0 \\ 0 & 1 & 0 \\ 0 & 0 & 1\end{array}\right]\left[\begin{array}{l}x_{1(k)} \\ x_{2(k)} \\ x_{3(k)}\end{array}\right]$

where, $x_{1}=a_{1} ; x_{2}=w_{1} ; x_{3}=\phi_{1} ;$

$y_{k}=x_{1 /(k)} \sin \left(k x_{2(k)} T_{s}+x_{3(k)}\right)+v_{k}$

$v_{k}=$ measurement noise

The above non-linear process can be represented as:

$x_{k+1}=x_{k}$

$y_{k+1}=G\left(x_{k}\right)+v_{k}$

Linearising the above system and applying the extended Kalman filter to the first order system, a non-linear recursive filter for estimating a single sinusoid and its frequency in white noise is obtained as follows [7]:

$$
\begin{aligned}
& \hat{x}_{k / k}=\hat{x}_{k / k-1}+K_{k}\left(y_{k}-G\left(\hat{x}_{k / k-1}\right)\right) \\
& \hat{x}_{k+1 / k}=\hat{x}_{k / k} \\
& K_{k}=\left.\hat{P}_{k / k-1} \frac{\partial G^{T}}{\partial X}\right|_{(k, k-1)} \\
& {\left[\left.\left.\frac{\partial G}{\partial X}\right|_{(k, k-1)} \hat{P}_{k / k-1} \frac{\partial G}{\partial X}\right|_{(k, k-1)}\right]^{-1}(\mathrm{~A}-9)} \\
& \hat{P}_{k / k}=\hat{P}_{k / k-1}-\left.K_{k} \frac{\partial G}{\partial X}\right|_{(k, k-1)} \hat{P}_{k / k-1} \text { (A-10) } \\
& \hat{P}_{k / k+1}=\hat{P}_{k / k}
\end{aligned}
$$

The initial estimate of the covariance matrix matrix $P$ is decided by trial and error and fixed to an identity matrix. The covariance resetting under abnormal changing conditions is decided by the same hysteresis method discussed earlier. The filter is non-linear and therefore the gain $K_{k}$ and the covariance matrix $\hat{P}_{k / k}$ depend on the estimate $\hat{x}_{k / k}$ of the state vector $x_{k}$.

\section{REFERENCES}

[1]. David Hart et.al "A new frequency tracking and plaasor estimation algorithm for generator protection", IEEE Trans. om Power Delivery, Vol.12, No.3. July 1997

[2]. Joe. F. Chucliaro et.al "Power System harmonic signal estumation and retrıval for active power filter applications", IEEE Trans. on Power Electronics, Vol.9, No.6, Nov.1994, PP580-585.

[3]. Moore et.al. "Model system tests on a new numeric method of power system frequency measurernent", LEEE Trans. on Power Delivery, Vol.11, No 2, April 1996.

[4]. Barbara F. La Scala et.al. "Design of an extended Klaman filter frequency tracker", IEEE Trans. on Signal Procesing, Vol.45, Ni.6, June 1997, pp 1473. 1484.

[5]. Petr Tichavsky et.al. "Comparative study of four adaptive frequency trackers", IEEE Trans on Signal Processing, Vol.45, Ni.6. June 1997. pp. $1473-1484$

[6]. Kıyoshi Nishiyama "A non-linear filter for estimating a sinusoidal signal and its parameters in white noise: on the case of a single sinusoid", IEEE Trans on Signal Processing, Vol.45, No.4, April 1997, pp970-981

[7] C.K. Chui, G. Chen " Klaman filtering with real-time applications" Springers Verlag $2^{\text {nd }}$ edition. 
Signal $=A \cos \left(k \omega T_{s}+\phi_{1}\right)+$ noise
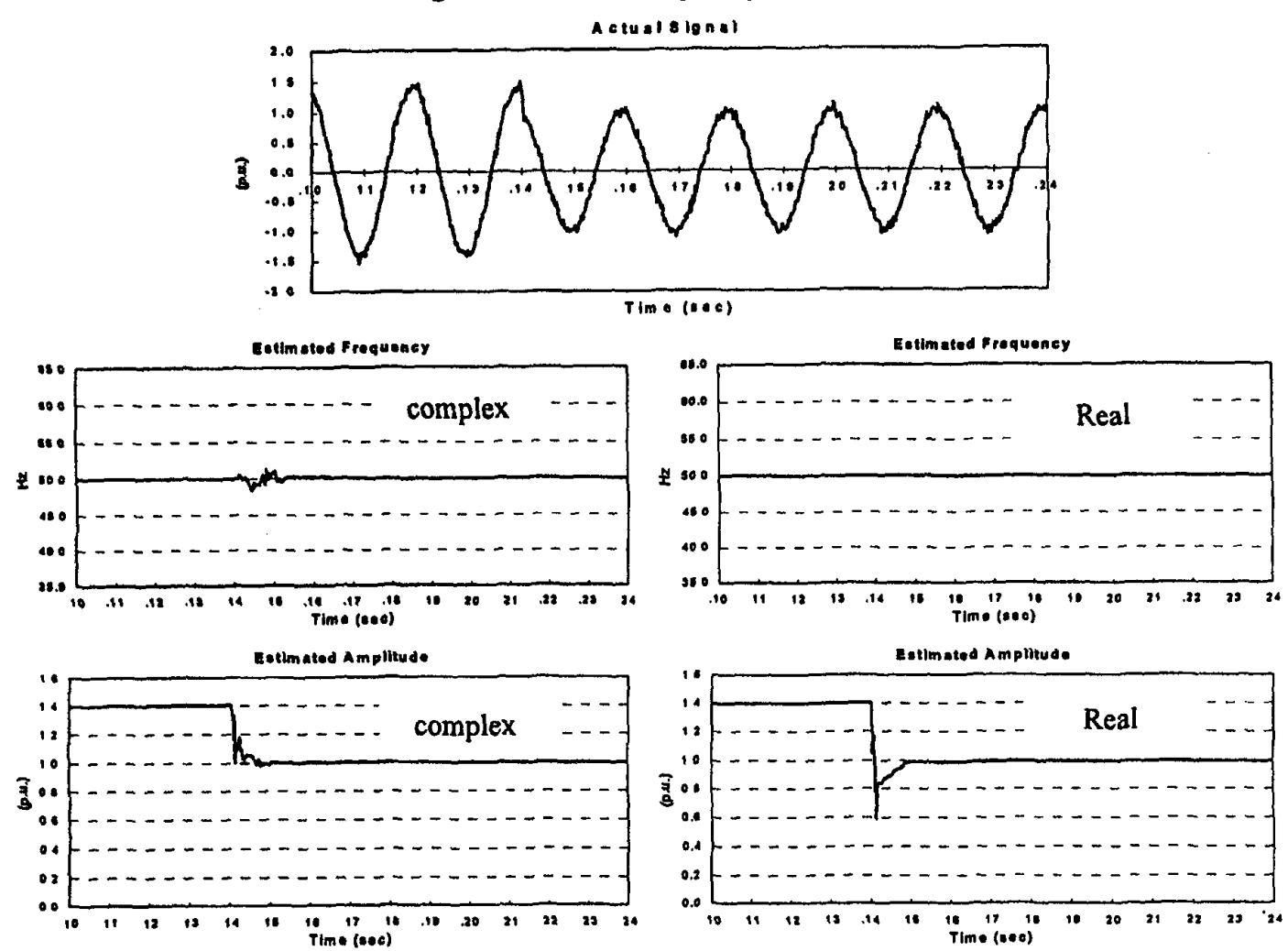

Fig.2 Step down in the amplitude

Signal $=A \cos \left(k \omega T_{s}+\phi_{1}\right)+\frac{A}{10} \cos \left(3 k \omega T_{s}+\phi_{3}\right)+\frac{A}{20} \cos \left(5 k \omega T_{s}+\phi_{5}\right)+$ noise
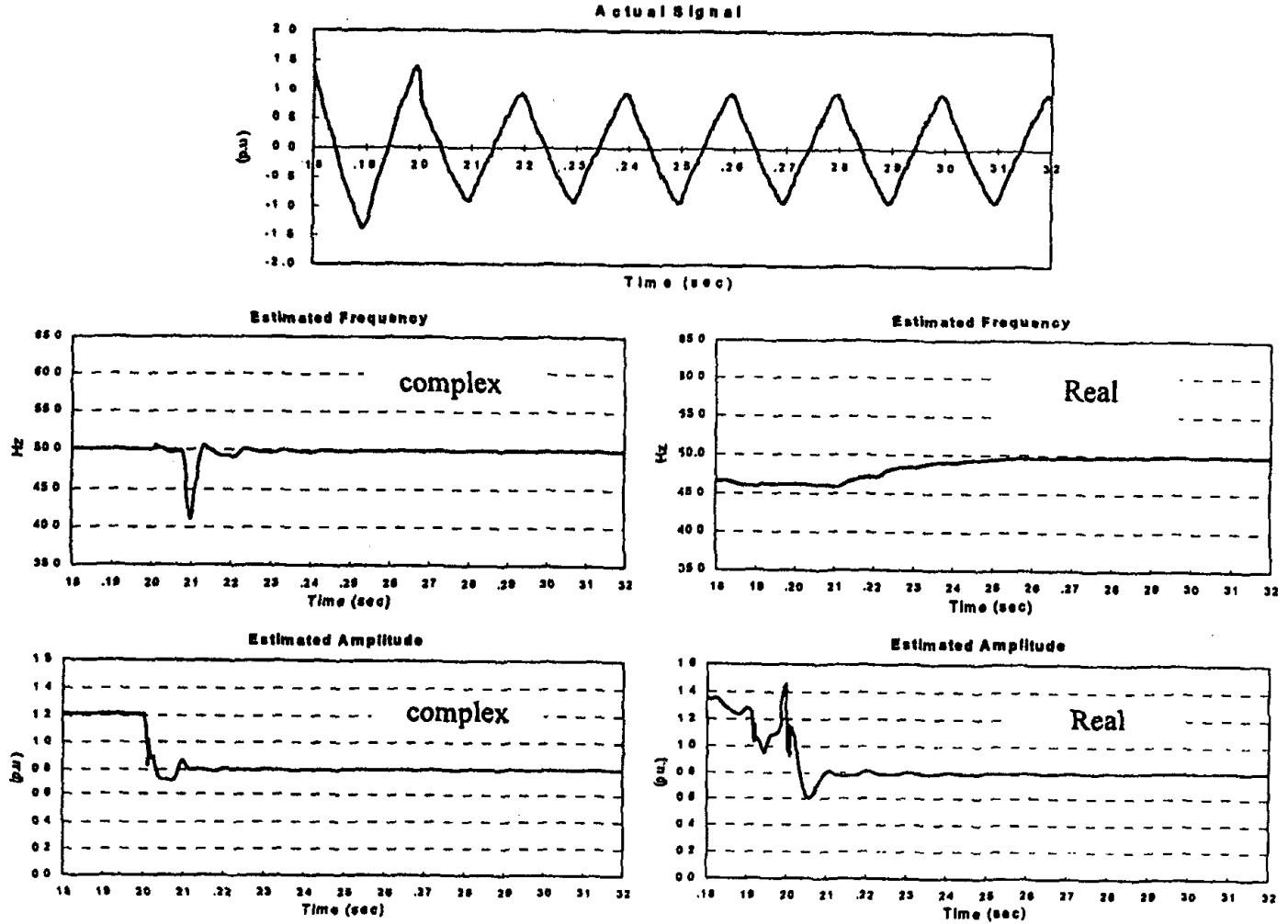

Fig. 3 Step down in the amplitude 
Signal $=A \cos \left(k \omega T_{s}+\phi_{1}\right)+\frac{A}{10} \cos \left(3 k \omega T_{s}+\phi_{3}\right)+\frac{A}{20} \cos \left(5 k \omega T_{s}+\phi_{s}\right)+$ noise
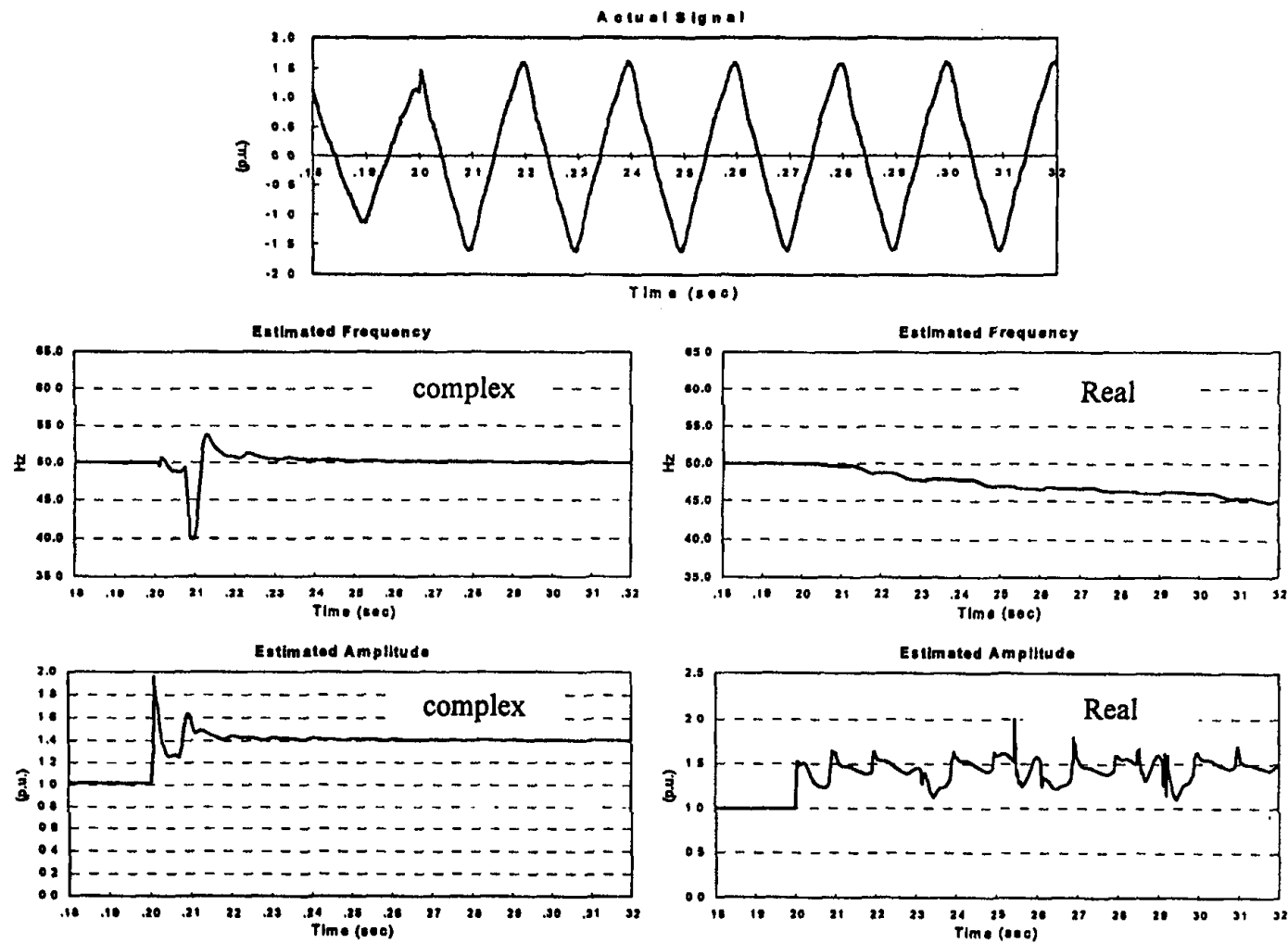

Fig. 4 Step up in the anplitude Signal $=A \cos \left(k \omega T_{s}+\phi_{1}\right)+$ noise
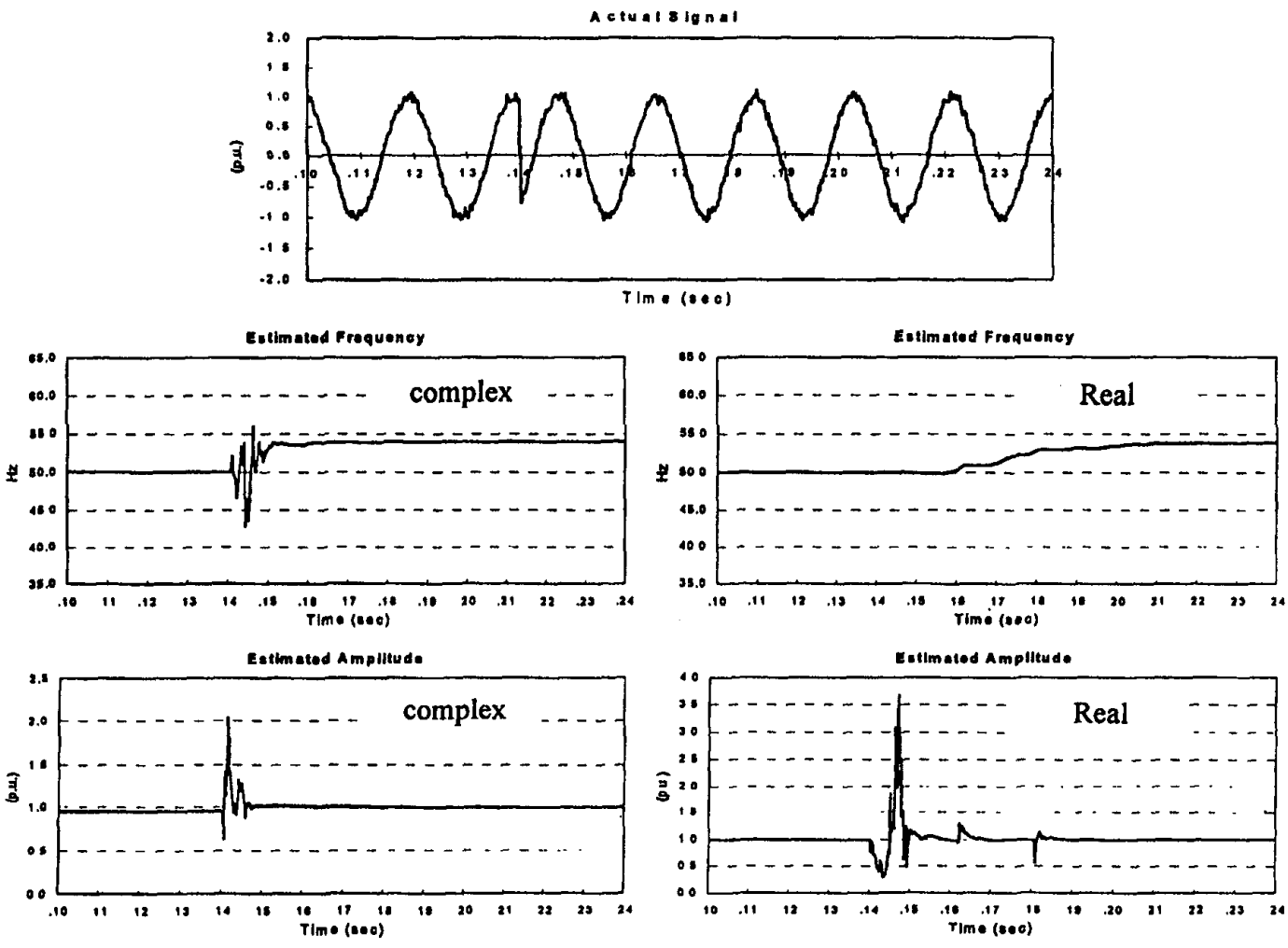

Fig. $58 \%$ Step up in the frequency 


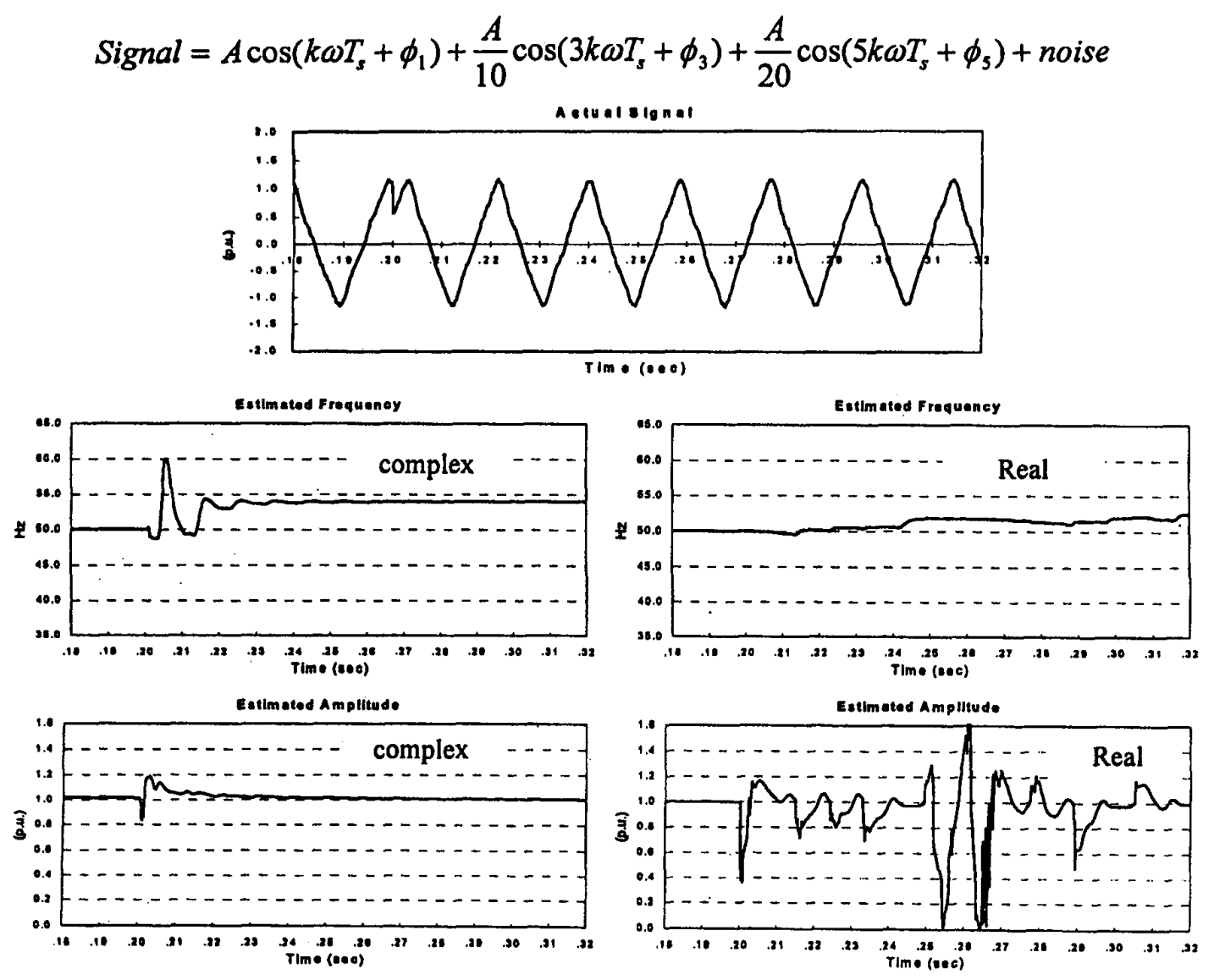

Fig. $6 \%$ Step up in the frequency

Signal $=A \cos \left(k \omega T_{s}+\phi_{1}\right)+$ noise
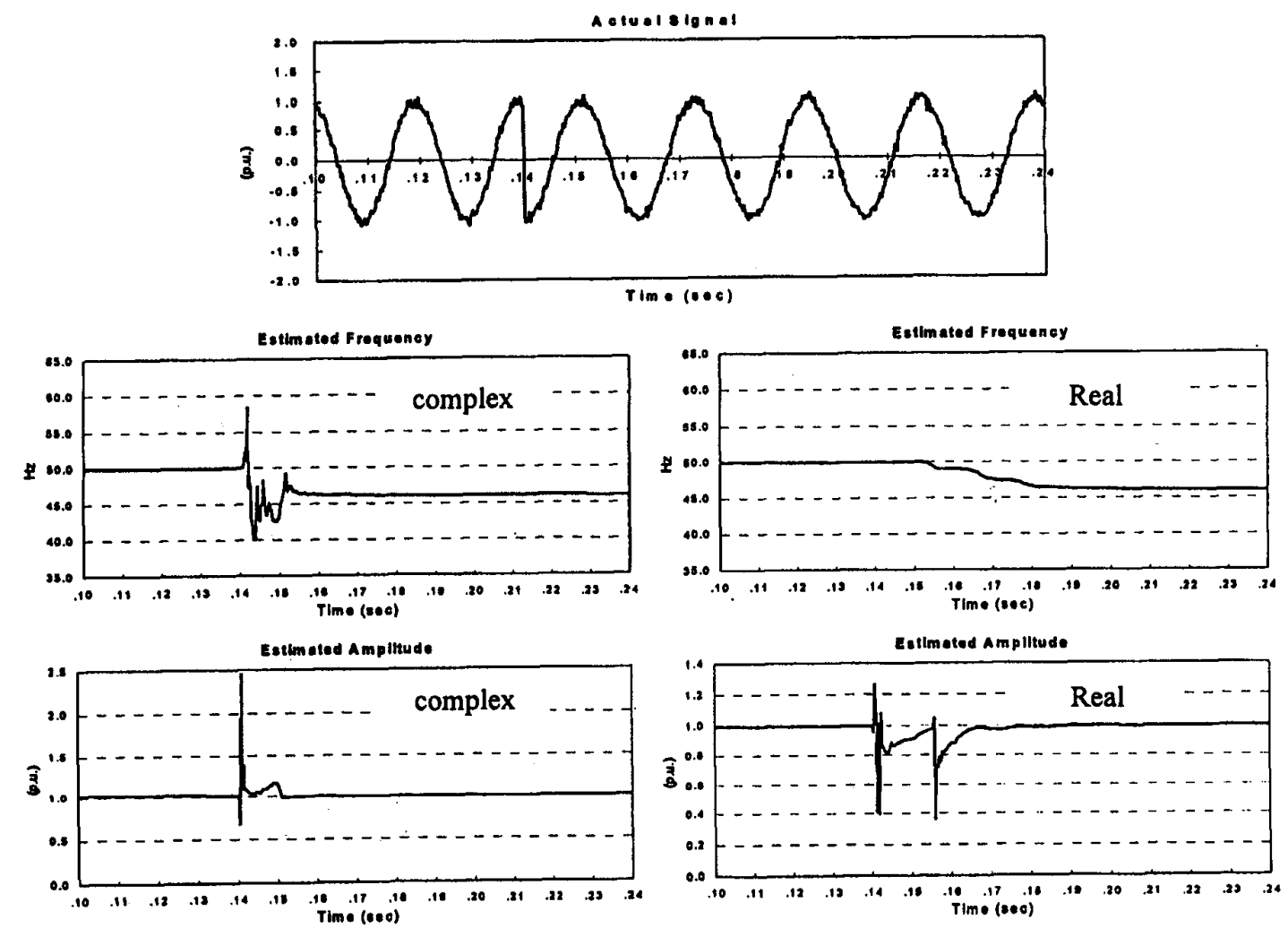

Fig. 7 Step down in the frequency 